\title{
Non-Hodgkin's lymphoma in a hemophilic patient with a traumatic hematoma
}

\section{Travmatik hematomu olan hemofilik hastada non-Hodgkin lenfoma}

\author{
Nihal Özdemir ${ }^{1}$, Ayşe Yurt ${ }^{1}$ Emine Türkan², Tiraje Celkan ${ }^{1}$ \\ ${ }^{1}$ Department of Pediatric Hematology, Cerrahpaşa Faculty of Medicine, İstanbul University, İstanbul, Turkey \\ ${ }^{2}$ Department of Pediatric Hematology Oncology, Okmeydanı State Hospital, Istanbul, Turkey
}

\section{To the editor,}

Hemophilia A, the most common severe inherited bleeding disorder, is characterized by FVIII deficiency. The use of recombinant FVIII products and improved purity of plasma-derived concentrates have decreased the risk of HIV, and hepatitis $\mathrm{B}$ and $\mathrm{C}$ virus transmission in patients with hemophilia. Malignancies reported in hemophilic patients are usually associated with $\mathrm{HIV}$, and hepatitis B and C infection. The incidence of non-infection-related malignancy is not known due to the limited quantity of data in the literature [1]. Herein we report a boy with hemophilia A that was followed-up with the initial diagnosis of a neck hematoma; examination of a subsequent excisional biopsy specimen resulted in the diagnosis of nonHodgkin's lymphoma (NHL).

A 12-year-old boy with mild hemophilia A (FVIII coagulant activity 7\%; vWF Ag: 78\%; Ricof: 72\%) had been followed-up at another center since he was 5 years old. Six months before presentation at our institution the patient had a tooth extracted and developed a mass in the neck. Initially, a muscle hematoma was suspected. As the mass enlarged, needle aspiration was performed, and the result showed reactive hyperplasia. The patient was referred to our clinic for further investigation.

The patient did not have symptoms suggesting lymphoma or any other malignancy. Physical examination was normal, except for a $2 \times 1.5$-cm soft mass in the neck. Full blood count, peripheral blood smear, sedimentation rate $(7 \mathrm{~mm} / \mathrm{h})$, and lactate dehydrogenase (LDH: 184 IU/L) were normal. Cervical magnetic resonance imaging (MRI) showed a heterogenous solid mass between the submandibular gland and sternocleidomastoid muscle. Thoracic and abdominal computed tomography (CT) results were normal. Positron emission tomography (PET) showed hypermetabolic lymphadenopathies in the left inferior cervical and supraclavicular fossa. Excisional biopsy under factor replacement showed T cell-rich diffuse B cell NHL. There was no bone marrow or cerebrospinal fluid involvement, and he was classified as stage 2 NHL. Viral serology, including HIV, and hepatitis $\mathrm{B}$ and $\mathrm{C}$ viruses was negative. The patient was treated according to the BFM-NHL 95 protocol and did not have any severe bleeding episodes during chemotherapy. Moreover, no complications occurred during cerebrospinal fluid tap or bone marrow aspiration/biopsy. 
Cerebrospinal fluid tap was performed 4 times by an experienced hematologist every time, and FVIII infusion and fresh frozen plasma were not administered. At the end of therapy, PET-CT was normal and the patient has been in remission for 1 year, as of the time this report was written.

Most malignancies in hemophilic patients are related to HIV and hepatitis infections due to contaminated blood products. Walker and Julian analyzed the cause of death in Canadians with hemophilia between 1980 and 1995, and reported a marked increase in deaths from liver cancer and lymphoma in patients with HCV or HIV infections, as compared to the general population [2]. A study performed in the UK between 1978 and 1999 with a hemophilic population reported 67 NHL cases in HIV-positive patients [3], whereas the incidence was much lower in HIV-negative individuals only 9 cases. The number of cases of NHL among HIVnegative hemophilic patients was equal to the number of cases expected in the general population. There are also reports of HIV-negative patients with Hodgkin's lymphoma [4,5].

Hemophilia may influence the clinical presentation and diagnostic evaluation of malignancies. As the presented case had hemophilia and a history of trauma, there was a delay in the diagnosis of lymphoma. A similar case with severe hemophilia and Hodgkin's disease also highlights the importance of clinical awareness when the signs and symptoms of a presumed hematoma do not resolve with time [5]. There are also case reports of patients with hemophilia that were eventually diagnosed with soft tissue sarcomas that mimicked pseudotumors [6].

Another important issue is the difficulty in treatment caused by the coexistence of bleeding diathesis and malignancy. Specific clinical problems may emerge during chemotherapy in patients with hemophilia and cancer, which may be challenging for physicians. The inherent tendency for bleeding may increase the probability of bleeding due to thrombocytopenia caused by chemotherapy. Invasive procedures, such as bone marrow biopsy or catheter replacement, may require factor replacement. Chemotherapy-induced cytopenia, mucosal toxicity, and hemostatic disturbances may be more problematic than expected. There are only few case reports on the management of leukemia in hemophilic patients [7-9]. A study of 2 patients by Zulfikar reported the existence of social and psychological burden associated with the coexistence of 2 difficult diseases, such as acute leukemia and severe hemophilia, among patients and families [7]. Based on our experience managing such cases, we recommend regular and adequate factor replacement in order to minimize the risk of bleeding during treatment. As the presented case had mild hemophilia, there were no major problems during his treatment.

In conclusion, the management of cancer in hemophilic patients is challenging. Consideration of probable malignancy in the differential diagnosis of patients with hemophilia and posttraumatic hematoma is critical. Additional research on the management of hemophilic patients with cancer may result in improved treatment options. Written informed consent was obtained from the patient.

\section{Conflict of interest statement}

The authors of this paper have no conflicts of interest, including specific financial interests, relationships, and/or affiliations relevant to the subject matter or materials included.

\section{References}

1. Dunn AL. Malignancy in patients with hemophilia: a review of the literature. Hemophilia 2010;16:427-36.

2. Walker IR, Julian JA. Causes of death in Canadians with hemophilia 1980-1995. Association of Hemophilia Clinic Directors of Canada. Hemophilia 1998;4:714-20.

3. Wilde JT, Lee CA, Darby SC, Kan SW, Giangrande P, Phillips AN, Winter M, Spooner R, Ludlam CA; UK Haemophilia Centre Doctors' Organisation. The incidence of lymphoma in the UK hemophilia population between 1978 and 1999. AIDS 2002;16:1803-7.

4. Koç A, Varan A, Büyükpamukçu M, Gürgey A. A case of hemophilia A associated with Hodgkin's disease. Turk J Pediatr 1999;41:517-20.

5. Smith R, Marson B, Smith M, Rangarajan S. Incidental finding of Hodgkin's disease following initial presentation with traumatic haematoma in the thoracic inlet. Hemophilia 2008;14:610-2.

6. Connor P, Cheung B, Rangarajan S. Malignant sarcoma masquerading as a pseudotumour in a patient with mild hemophilia. Br J Haematol 2003;123:191.

7. Zulfikar B. Two patients with hemophilia and acute leukaemia. Hemophilia 2002;8:698-702.

8. Altay C, Hicsonmez G, Zamani VP, Sagin G, Tuncer M, Ozsoylu S. Acute leukemia in two patients with hemophilia. Cancer 1985;55:510-1.

9. Clark C, Gidvani VK. Acute myelogenous leukemia in a patient with a severe factor IX deficiency. Pediatr Blood and Cancer 2011;56:156-7. 